\title{
BAIRE CATEGORY AND $B_{r}$-SPACES
}

\author{
DOMINIKUS NOLL
}

(Communicated by Dennis Burke)

\begin{abstract}
A topological space satisfying the open mapping theorem is called a $B_{r}$-space. We investigate the question whether completely regular $B_{r}$-spaces must be Baire spaces. The answer we obtain is twofold and surprising. On the one hand there exist first category completely regular $B_{r}$-spaces. Examples are provided in the class of Lindelöf $P$-spaces. On the other hand, we obtain a partial positive answer to our question. We prove that every suborderable metrizable $B_{r}$-space is in fact a Baire space. We conjecture that this is true for metrizable $B_{r}$-spaces in general. Our paper is completed by some applications. For instance, we establish the existence of a metrizable $B_{r}$-space $E$ whose square $E \times E$ is no longer a $B_{r}$-space.
\end{abstract}

Introduction. A Hausdorff topological space $E$ is called a $B_{r}$-space (resp. a $B$ space) if every continuous, nearly open bijection (resp. surjection) $f$ from $E$ onto an arbitrary Hausdorff topological space $F$ is open.

Every locally compact Hausdorff space is a $B$-space and every $B$-space is a $B_{r^{-}}$ space. Every Cech complete space is known to be a $B_{r}$-space (see [BP]). $B_{r}$-spaces have been investigated in several papers; see [We, BP, $\mathbf{N}_{1}, \mathbf{N}_{2}$ ].

In our paper $\left[\mathbf{N}_{2}\right.$ ] we have examined the problem of invariance of the class of $B_{r^{-}}$ spaces under the operation of taking finite sums. We have obtained the following result.

Let $E$ be a completely regular $B_{r}$-space. Then the following statements are equivalent:

(1) $E$ is a Baire space.

(2) Whenever $F$ is a Cech complete space, then the topological sum $E \oplus F$ is again a $B_{r}$-space.

We left open in $\left[\mathbf{N}_{2}\right]$ the question whether completely regular $B_{r}$-spaces are Baire-spaces in general, i.e., whether statement (2) above is true for arbitrary completely regular $B_{r}$-spaces $E$. In fact, one is tempted to conjecture that the answer to this question is in the positive if one takes into account the following facts:

(a) Given an arbitrary $B_{r}$-space $E$, the sum $E \oplus E$ is again a $B_{r}$-space (see $\left[\mathrm{N}_{2}\right.$, Theorem 1]), and the same is true for any sum $E \oplus L$, where $L$ is an arbitrary locally compact Hausdorff space. So why should not $E \oplus F$ be a $B_{r}$-space for arbitrary Čech complete spaces $F$ ?

(b) There is a partial positive answer to our question in the class of strongly zero-dimensional metrizable $B_{r}$-spaces (which we shall prove in the present paper) stating that every strongly zero-dimensional metrizable $B_{r}$-space is in fact a Baire space.

Received by the editors December 31, 1985 and, in revised form, August 11, 1986.

1980 Mathematics Subject Classification (1985 Revision). Primary 46A30, 02G20, 54C10. 
For all that, the answer to our question is in the negative in general. The counterexample we present is provided by an utterly pathological class of spaces. We prove that every Lindelöf $P$-space is a $B_{r}$-space and then give an example of a first category space of this type. Nevertheless, the question whether metrizable $B_{r^{-}}$ spaces are Baire spaces in general remains open. I conjecture that this is actually the case.

The remainder of our paper is devoted to some applications of the main results stated above. For instance we establish the existence of a strongly zero-dimensional metrizable $B_{r}$-space $E$ whose square $E \times E$ is no longer a $B_{r}$-space. The existence of such a counterexample is suggested by the situation in the categories of locally convex vector spaces and topological groups, where the corresponding counterexamples are known to exist (see [Kö, p. 31 and $\mathbf{G r}$ ]. Finally, we examine the class of $B$-spaces. We give an example of a completely metrizable space which is not a $B$-space. We prove that the sum of two $B$-spaces need not be a $B$-space in general and we prove the surprising fact that given a nondiscrete Lindelöf $P$-space $E$, the product $E \times E$ is a $B$-space while the product $E \times \beta E$ is not.

Our basic terminology is from the book [E]. A mapping $f: E \rightarrow F$ is called nearly open if for every $x \in E$ and every neighborhood $U$ of $x$ the set $\overline{f(U)}$ is a neighborhood of $f(x)$.

1. Metrizable $B_{r}$-spaces. All metrizable $B_{r}$-spaces known up to now are Baire spaces. I conjecture that this is true in general, but in the present paper we only obtain a partial positive answer in the framework of strongly zero-dimensional metrizable spaces and, as a consequence, in the case of suborderable metrizable spaces.

Before stating our first result we recall that a topological space is called strongly zero-dimensional if it has dimension zero with respect to the covering dimension dim.

PROPOSITION 1. Every strongly zero-dimensional metrizable $B_{r}$-space $E$ is a Baire space.

Proof. For $X \subset E$ we denote by $w(X)$ the weight of the subspace $X$. Every nonempty open subset $U$ of $E$ contains some clopen nonempty subset $V$ which is minimal with respect to $w$ in the sense that $w(V)=w(O)$ holds for every nonempty clopen subset $O$ of $V$. On the other hand, every clopen subspace $V$ of $E$ is itself a $B_{r}$-space. So it is sufficient to prove that every strongly zero-dimensional metrizable $B_{r}$-space $E$ which is minimal with respect to $w$ in the sense above is of the second category. So let $E$ be of this type.

Assume that $E$ is of the first category and choose a sequence $G_{1}, G_{2}, \ldots$ of dense open subsets of $E$ with $G_{n+1} \subset G_{n}$ having $\bigcap\left\{G_{n}: n \in \mathbf{N}\right\}=\varnothing$. There exists a sequence $\left(\mathfrak{V}_{n}\right)_{n=1}^{\infty}$ of open covers of $E$ with the following properties:

(1) Each $\mathfrak{V}_{n}$ is disjoint, i.e. $V \cap V^{\prime}=\varnothing$ for $V, V^{\prime} \in \mathfrak{V}_{n}, V \neq V^{\prime}$.

(2) $\varnothing \notin \mathfrak{V}_{n}$ and $\mathfrak{V}_{n+1}$ refines $\mathfrak{V}_{n}$.

(3) Each $V \in \mathfrak{V}_{n}$ has diameter $<1 / n$ (with respect to some fixed metric for $E$ ).

(4) $\operatorname{card}\left\{V \in \mathfrak{V}_{1}: V \not \subset G_{1}\right\}=\operatorname{card}\left\{V \in \mathfrak{V}_{1}: V \subset G_{1}\right\}$.

(5) For every $n \in \mathbf{N}$ and every $V \in \mathfrak{V}_{n}$

$\operatorname{card}\left\{W \in \mathfrak{V}_{n+1}: W \subset V, W \not \subset G_{n+1}\right\}=\operatorname{card}\left\{W \in \mathfrak{V}_{n+1}: W \subset V, W \subset G_{n+1}\right\}$. 
Note that $\left(\mathfrak{V}_{n}\right)$ may be found satisfying (4) and (5) in view of the imposed minimality with respect to the weight function $w$. Now by induction we define mappings ${ }^{*}: \mathfrak{V}_{n} \rightarrow \mathfrak{V}_{n}, n=1,2, \ldots$, such that the following conditions are satisfied:

(a) $V^{* *}=V$ for each $V \in \mathfrak{V}_{n}$.

(b) $V \in \mathfrak{V}_{n}$ and $V \not \subset G_{n}$ implies $V^{*} \subset G_{n}$

(c) $V \in \mathfrak{V}_{n}, W \in \mathfrak{V}_{n+1}$, and $W \subset V^{*}$ imply $W^{*} \subset V$.

Let $\mathfrak{B}$ denote the set of all $\tilde{V}=V \cup V^{*}, V \in \mathfrak{V}_{n}, n \in \mathbf{N}$. We claim that $\mathfrak{B}$ is a basis for a new topology on $E$. Indeed, let $x \in \tilde{V} \cap \tilde{W}, V \in \mathfrak{V}_{n}, W \in \mathfrak{V}_{m}$, $n \geq m$. If $x \in V \cap W$, then $V \subset W$, hence $V^{*} \subset W^{*}$, giving $\tilde{V} \subset \tilde{W}$. Assume $x \in V \cap W^{*}$; then $V \subset W^{*}$, hence $V^{*} \subset W$, giving $\tilde{V} \subset \tilde{W}$ by condition (a). Next assume $x \in V^{*} \cap W$. This gives $V^{* *} \subset W^{*}$ by (c), hence $\tilde{V} \subset \tilde{W}$ by (a) again. Finally assume that $x \in V^{*} \cap W^{*}$. Again conditions (a) and (c) imply $\tilde{V} \subset \tilde{W}$. So $\mathfrak{B}$ generates a topology on $E$ which is strictly coarser than the original topology, in view of the fact that $V \neq V^{*}$ whenever $V \in \mathfrak{V}_{n}, V \not \subset G_{n}$.

Let $F$ denote the set $E$ with this new topology. We prove that $F$ is Hausdorff. Let $x, y \in E, x \neq y$. Assume that $y \in \operatorname{cl}_{F} \tilde{U}$ holds for every $U$ having $x \in U$. This provides a sequence $V_{1}, V_{2}, \ldots$ with $V_{n} \in \mathfrak{V}_{n}$ such that $x \in V_{n}, y \in V_{n}^{*}, n=1,2, \ldots$ But $x \in V_{n}$ implies $V_{n} \not \subset G_{n}$ for some $n_{0}$ and all $n \geq n_{0}$, hence $V_{n}^{*} \subset G_{n}$ holds for $n \geq n_{0}$ in view of condition (b). This yields $y \in G_{n}, n \geq n_{0}$, a contradiction. So $F$ is in fact a Hausdorff space.

Finally, we prove that the identity mapping id: $E \rightarrow F$ is nearly open. Since $E$ is a $B_{r}$-space, this implies $E=F$, a contradiction, as desired. To prove the claim it is sufficient to show that $\tilde{V} \subset \operatorname{cl}_{F} V$. Since $V \subset \operatorname{cl}_{F} V$ is clear, we prove $V^{*} \subset \operatorname{cl}_{F} V$. Let $x \in V^{*}$. Let $V \in \mathfrak{V}_{n}, m \geq n, x \in W \in \mathfrak{V}_{m}$. We have to prove that $\tilde{W}$ intersects $V$. But note that $W \subset V^{*}$ gives $W^{*} \subset V$ and this proves $\tilde{W} \cap V \neq \varnothing$.

A topological space $E$ is called suborderable if it is a subspace of some linearly orderable space (see $[\mathbf{L u}]$ ). Now we obtain the following

THEOREM 1. Every suborderable metrizable $B_{r}$-space $E$ is a Baire space.

ProOF. Following [Lu, 2.9], $E$ may be represented as a dense subspace of some compact linear ordered space $\tilde{E}$. Suppose that $E$ is not Baire. Then there exists a nonempty open subset $U$ of $E$ which is of the first category. Consequently, every nonempty open subset $V$ of $U$ must contain a gap, i.e. for $x, y \in V, x<y$ there exists $u \in \tilde{E} \backslash E$ such that $x<u<y$. Let $u, v$ with $u<v$ be fixed gaps with $[u, v] \cap E$ contained in $U$. Clearly $[u, v] \cap E$ is clopen in $E$ and so is $B_{r}$. Moreover, this space is strongly zero-dimensional since the gaps are dense in $[u, v]$. This proves that $[u, v] \cap E$ is of the second category in $E$.

In view of the theorem stated in the introduction (which is Theorem 2 in $\left[\mathbf{N}_{2}\right]$ ) we obtain the following

COROLlARY. Let $E$ be a suborderable metrizaule $B_{r}$-space and let $F$ be a Čech complete space. Then $E \oplus F$ is a $B_{r}$-space.

2. $P$-spaces. A topological space $E$ is called a $P$-space if every $G_{\delta}$ subset of $E$ is open. Some authors call this a quasi $P$-space and reserve the term $P$-space for completely regular spaces of this type. A topological space $E$ is called locally Lindelöf if every $x \in E$ admits a base of neighborhoods which are Lindelöf with their induced topologies. Now we can state the following theorem. 
THEOREM 2. Every Hausdorff locally Lindelöf $P$-space $E$ is a B-space.

ProOF. Let $f: E \rightarrow F$ be a continuous, nearly open surjection onto the Hausdorff space $F$. We may assume that $F$ is semiregular. Indeed, if $F_{s}$ denotes the semiregularization of $F$ then $F_{s}$ is Hausdorff and $f: E \rightarrow F_{s}$ is still continuous and nearly open. But under this assumption $F$ must be a $P$-space. For let $G$ be a $G_{\delta}$ set in $F, G=\bigcap\left\{G_{n}: n \in \mathbf{N}\right\}$ for open sets $G_{n}$ and let $y \in G, y=f(x)$ for some $x$. Now for every $n$ there exists an open set $V_{n}$ with $x \in V_{n}$ and with int $\overline{f\left(V_{n}\right)} \subset G_{n}$. Now $V=\bigcap\left\{V_{n}: n \in \mathbf{N}\right\}$ is open in $E$ and clearly we have int $\overline{f(V)} \subset G_{n}$ for every $n$, which proves that $y$ is an interior point of $G$.

Now let $x \in E$ and some Lindelöf neighborhood $U$ of $x$ be fixed. We show that $f(U)$ is closed in $F$. Assume the contrary and let $y \in \overline{f(U)} \backslash f(U)$. Let $\mathfrak{F}$ be the filter of neighborhoods of $y$. Now $\{f(U) \backslash \bar{A}: A \in \mathfrak{F}\}$ is an open cover of $f(U)$ and consequently has a countable subcover, say $f(U)=\bigcup\left\{f(U) \backslash \bar{A}_{n}: n \in \mathbf{N}\right\}$ for $A_{n} \in \mathfrak{F}$. But this yields $\bigcap\left\{\bar{A}_{n}: n \in \mathbf{N}\right\} \cap f(U)=\varnothing$, a contradiction since we have $\bigcap\left\{A_{n}: n \in \mathbf{N}\right\} \in \mathfrak{F}$. This proves that $f(U)$ is closed. But now we have $f(x) \in \operatorname{int} \overline{f(U)}=\operatorname{int} f(U)$, hence $f$ must be open.

REMARKS. (1) Every Hausdorff locally Lindelöf $P$-space is completely regular (see [Ca, Corollary 7.4]). This is why we did not use the term quasi $P$-space here.

(2) The first part of the proof of Theorem 2 may be used to prove that every Hausdorff minimal $P$-space is a $B$-space. Note that Hausdorff minimal $P$-spaces need not be regular in general (see [Ca, Example 9.1]), but are semiregular as a consequence of the fact that every $B_{r}$-space is semiregular.

(3) Theorem 2 may easily be generalized to higher cardinalities. If $E$ is called a $P_{\kappa}$ space in case the intersection of any family $\left\{G_{\alpha}: \alpha<\kappa\right\}$ of open sets is open, then every Hausdorff $P_{\kappa}$-space with the property that every open cover has a subcover of cardinality $\leq \kappa$ is in fact a $B$-space.

(4) Hausdorff Lindelöf $P$-spaces may be obtained by the following process. Let $E$ be a compact scattered space and let $E^{\delta}$ denote the set $E$ with the $G_{\delta}$-topology, i.e., the topology generated by the $G_{\delta}$ sets in $E$. Then $E^{\delta}$ is a Lindelöf $P$-space.

EXAMPLE 1. We are going to construct a first category Lindelöf $P$-space. Let $F$ denote the set of all ordinals $\alpha \leq \omega_{1}$ and let $\{\alpha\},\left(\alpha, \omega_{1}\right]$ be open sets in $F$ for $\alpha<\omega_{1}$. Clearly $F$ is a Lindelöf $P$-space. Let $F^{\mathbf{N}}$ have the box-product topology. Again $F^{\mathbf{N}}$ is a $P$-space. Finally let $E$ denote the subspace of $F$ consisting of all $f \in F^{\mathbf{N}}$ with $f(n)=\omega_{1}$ for almost all $n$. Clearly $E$ is a $P$-space. We claim that $E$ is also Lindelöf. Indeed, let $E(n)$ denote the set of $f \in F^{\mathbf{N}}$ with $f(k)=\omega_{1}$ for all $k \geq n$. We have $E=\bigcup\{E(n): n \in \mathbf{N}\}$. It is therefore sufficient to prove that all $E(n)$ are Lindelöf. But note that $E(1)$ is a one-point space while we have $E(n) \cong F^{n-1}$ for $n \geq 2$, which proves that $E(n)$ is Lindelöf since finite products of Lindelöf $P$-spaces are known to be Lindelöf $P$. Finally note that $E$ must be of the first category since it is the union of the closed nowhere dense subsets $E(n)$, $n \in \mathbf{N}$. Clearly $E$ is a Hausdorff space. This yields the desired counterexample. Note that $E$ being Lindelöf and regular it is also paracompact.

EXAMPLE 2. Let $E$ be the space constructed in Example 1. Then there exists a Cech complete space $F$ such that $E \oplus F$ is not a $B_{r}$-space. We may choose $F$ to be any dense $G_{\delta}$ subset of $\beta E$ having $E \cap F=\varnothing$. Of course the existence of $F$ may as well be deduced from our theorem stated in the introduction. 
3. Products of $B_{r}$-spaces. In $\left[\mathbf{N}_{2}\right]$ we have provided examples of strongly zero-dimensional metrizable $B_{r}$-spaces $E, F$ whose product $E \times F$ is no longer a $B_{r}$-space. In this section we obtain a stronger negative result, i.e., we prove the existence of a strongly zero-dimensional metrizable $B_{r}$-space $E$ whose square $E \times E$ is no longer $B_{r}$.

EXAMPLE 3. There exists a strongly zero-dimensional metrizable $B_{r}$-space $E$ whose square $E \times E$ is a Baire space but whose cube $E \times E \times E$ is of the first category.

Our construction is based on an example given by Fleissner and Kunen (see [FK, Ex. 4]). Let $c=2^{\omega}$ and let $C_{\omega} c^{+}$denote the set of ordinals $\alpha<c^{+}$with $c f(\alpha) \leq \omega$, where $c^{+}$denotes the successor-cardinal of $c$. Let $A_{y}, y \in 3^{\omega}$, be mutually disjoint stationary subsets of $C_{\omega} c^{+}$. Recall that a subset $S$ of a cardinal $\kappa$ is called stationary if it intersects every closed and cofinal subset $C$ of $\kappa$, where closed refers to the order-topology on $\kappa$. Note that $C_{\omega} c^{+}$itself is a stationary subset of $c^{+}$. For $x \in 3^{\omega}$ define a stationary subset $B_{x}$ of $C_{\omega} c^{+}$by

$$
B_{x}=\bigcup\left\{A_{y}: y \in 3^{\omega}, y(n) \neq x(n) \text { for all } n\right\} \text {. }
$$

Let 3 and $c^{+}$each be discretely topologized and let $3^{\omega} \times\left(c^{+}\right)^{\omega}$ have the product topology. Let $E$ denote the set of all $(x, f) \in 3^{\omega} \times\left(c^{+}\right)^{\omega}$ having $f^{*}=\sup \{f(n): n \in$ $\omega\} \in B_{x}$. Then $E$ is a strongly zero-dimensional metrizable space since it is a subspace of $3^{\omega} \times\left(c^{+}\right)^{\omega}$. It follows from [FK] that $E \times E$ is a Baire space while $E \times E \times E$ is not. It therefore remains to prove that $E$ is a $B_{r}$-space.

For $\sigma=\left(\left(\alpha_{0}, \beta_{0}\right), \ldots,\left(\alpha_{r}, \beta_{r}\right)\right) \in 3^{r+1} \times\left(c^{+}\right)^{r+1}$ we denote by $B(\sigma)$ the set of $(x, g) \in E$ having $x(i)=\alpha_{i}, g(i)=\beta_{i}$ for $i \leq r$. The sets $B(\sigma)$ form a basis for $E$. Let $F S$ denote the set of all such finite sequences $\sigma$.

Let $f: E \rightarrow F$ be a continuous, nearly open bijection onto some Hausdorff space $F$. Let $\left(x_{0}, g_{0}\right) \in E$ and a neighborhood $U=B\left(\sigma_{0}\right)$ of $\left(x_{0}, g_{0}\right)$ with $\sigma_{0}=\left(x_{0}, g_{0}\right) \uparrow$ $n$ be fixed. It is sufficient to prove that $f(U)$ is closed in $F$, for then int $\overline{f(U)}=$ int $f(U)$ proves the openness of $f$. Let $z_{0} \in \overline{f(U)}, z_{0}=f\left(y_{0}, h_{0}\right)$. Since $U$ is closed, it is sufficient to prove $U \cap V \neq \varnothing$ for an arbitrary neighborhood $V$ of $\left(y_{0}, h_{0}\right)$. We may choose $V$ of the form $V=B\left(\tau_{0}\right)$ with $\tau_{0}=\left(y_{0}, h_{0}\right) \uparrow m$. Consider any fixed well-order on FS. We define two mappings $\Theta_{1}, \Theta_{2}:$ FS $\times$ FS $\rightarrow$ FS along the following rules.

(1) $\Theta_{1}(\sigma, \tau)=\rho$ if $\rho$ is the first (for the well-order of FS) in FS with $B(\rho)$ contained in $f^{-1}$ (int $\left.\overline{f(B(\sigma))} \cap f(B(\tau))\right)$ and $\Theta_{1}(\sigma, \tau)=\tau$ if no such $\rho$ exists.

(2) $\Theta_{2}(\sigma, \tau)=\rho$ if $\rho$ is the first (for the well-order of FS) in FS with $B(\rho)$ contained in $f^{-1}$ (int $\left.\overline{f(B(\tau))} \cap f(B(\sigma))\right)$ and $\Theta_{2}(\sigma, \tau)=\sigma$ if no such $\rho$ exists.

Since $f$ is injective and $B\left(\rho_{1}\right) \subset B\left(\rho_{2}\right)$ implies $\rho_{1} \supset \rho_{2}$, we deduce that $\Theta_{1}(\sigma, \tau)$ $\supset \tau$ and $\Theta_{2}(\sigma, \tau) \supset \sigma$ hold for all $\sigma, \tau$ in FS.

Let $W$ denote the set of all ordinals $\alpha$ having $2<\alpha<c^{+}$with the following property:

There exist sequences $\left(\sigma_{n}\right)$ and $\left(\tau_{n}\right)$ such that $\Theta_{1}\left(\sigma_{0}, \tau_{0}\right)=\tau_{1}, \tau_{2} \supset \tau_{1},\left|\tau_{2}\right|=$ $\left|\tau_{1}\right|+1, \Theta_{2}\left(\sigma_{0}, \tau_{2}\right)=\sigma_{1}, \sigma_{2} \supset \sigma_{1},\left|\sigma_{2}\right|=\left|\sigma_{1}\right|+1$, etc. with $\bigcup_{n} \sigma_{n}=(x, g)$, $\bigcup_{n} \tau_{n}=(y, h)$, and $g^{*}=h^{*}=\alpha$. (Here $\sigma_{0}, \tau_{0}$ have the meaning defined above.)

For $z \in 3^{\omega}$ let $W_{z}$ denote the set of all ordinals $\alpha \in W$ for which $(x, g)$ and $(y, h)$ exist as above with $g^{*}=h^{*}=\alpha$ and with $z(n) \notin\{x(n), y(n)\}$ for all $n$. Then we have $W=\bigcup\left\{W_{z}: z \in 3^{\omega}\right\}$. 
We claim that $W$ is stationary in $c^{+}$. Indeed, let $C$ be closed and cofinal in $c^{+}$. Define $\tau_{2}, \tau_{4}, \ldots$ and $\sigma_{2}, \sigma_{4}, \ldots$ such that

$$
\left|\tau_{2 n}\right|=\left|\tau_{2 n-1}\right|+1, \quad\left|\sigma_{2 n}\right|=\left|\sigma_{2 n-1}\right|+1
$$

and

$$
\begin{aligned}
\sup \sigma_{2 n-1} & \leq \sup \sigma_{2 n} \in C, \quad \sup \tau_{2 n-1} \leq \sup \tau_{2 n} \in C, \\
& \sup \sigma_{2 n} \leq \sup \tau_{2 n+2} \leq \sup \sigma_{2 n+2},
\end{aligned}
$$

where in case $\rho=\left(\left(\alpha_{0}, \gamma_{0}\right), \ldots,\left(\alpha_{r}, \gamma_{r}\right)\right)$ sup $\rho$ denotes $\sup _{i} \gamma_{i}$. Then we have $\bigcup_{n} \sigma_{n}=(x, g)$ and $\bigcup_{n} \tau_{n}=(y, h)$ with $g^{*}=h^{*} \in C \cap W$. This proves that $W$ is stationary.

But note that $W$ cannot be the union of $2^{\omega}$ nonstationary subsets, hence some $W_{z}$ must be stationary as well. Now we shall use the following lemma, which is Lemma 1 in $[\mathbf{F K}]$.

LEMMA. If $K \subset\left(c^{+}\right)^{\omega}$ is closed and $W=\left\{f^{*}: f \in K\right\}$ is stationary, then there exists a closed cofinal subset $C$ of $c^{+}$such that $C \cap C_{\omega} c^{+} \subset W$.

Hence there exists a closed cofinal set $C$ in $c^{+}$with $C \cap C_{\omega} c^{+} \subset W_{z}$. Note that the lemma may be applied if we define the set $K$ consisting of all $((x, g),(y, h))$ with $\bigcup_{n} \sigma_{n}=(x, g), \bigcup_{n} \tau_{n}=(y, h), g^{*}=h^{*}$ for sequences $\left(\sigma_{n}\right),\left(\tau_{n}\right)$ as in the definition of $W$ such that, in addition, $z(n) \notin\{x(n), y(n)\}$ holds for all $n$. Indeed, $K$ is closed in $3^{\omega} \times\left(c^{+}\right)^{\omega} \times 3^{\omega} \times\left(c^{+}\right)^{\omega} \simeq\left(c^{+}\right)^{\omega}$ and we have $W_{z}=\left\{\psi^{*}: \psi \in K\right\}$.

Since $A_{z}$ is stationary in $C_{\omega} c^{+}$, we have $W_{z} \cap A_{z} \neq \varnothing$. Let $\alpha \in W_{z} \cap A_{z}$ and let $\left(\sigma_{n}\right),\left(\tau_{n}\right)$ be sequences as in the definition of $W_{z}$ with $\bigcup_{n} \sigma_{n}=(x, g), \bigcup_{n} \tau_{n}=$ $(y, h), g^{*}=h^{*}=\alpha, z(n) \notin\{x(n), y(n)\}$ for all $n$. $\alpha \in A_{z}$ implies $(x, g),(y, h) \in E$. On the other hand we have $(x, g) \in U,(y, h) \in V$, and $f(x, g) \in \operatorname{int} \overline{f\left(B\left(\tau_{n}\right)\right)}$ for all $n$. This proves $(x, g)=(y, h)$ and hence $U \cap V \neq \varnothing$ is proved.

EXAMPLE 4 . Let $E$ be the strongly zero-dimensional metrizable $B_{r}$-space constructed in Example 3. If $E \times E$ is not a $B_{r}$-space, we have found the desired counterexample. Otherwise $F=E \times E$ is a Baire space in view of Proposition 1 . But then $F$ is the desired counterexample, for $F \times F$ is of the first category since $E \times E \times E$ is, and hence cannot be a $B_{r}$-space in view of Proposition 1. So we have established the existence of a $B_{r}$-space whose square is no longer $B_{r}$.

Using Example 4 from [FK], one may construct for every $n<\omega$ a strongly zerodimensional metrizable space $E$ such that $E^{n}$ is a $B_{r}$-space but $E^{n+2}$ is not since it is of the first category. We do not know, however, whether in these cases $E^{n+1}$ is $B_{r}$ or not. In particular we do not know whether $E \times E$ must be a Baire space in case $E$ is a strongly zero-dimensional metrizable $B_{r}$-space.

4. $B$-spaces. It has been an open question for a long time whether every $B_{r^{-}}$ complete locally convex vector space was $B$-complete. Finally, this question has been answered in the negative by Valdivia (see [V]). In the category of topological groups the situation is comparatively simple. A $B_{r}$-group which is not a $B$-group may be found in [Su]. In the topological case the gap between $B$ - and $B_{r}$-spaces is rather wider. The following example shows that even completely metrizable space need not be $B$-spaces.

EXAMPLE 5. Let $E=\mathbf{R} \backslash \mathbf{Q} \oplus \mathbf{R}, F=\mathbf{R}$ and define $f: E \rightarrow F$ by $f \mid \mathbf{R} \backslash \mathbf{Q}=e_{\mathbf{R} \backslash \mathbf{Q}}$, $f \mid \mathbf{R}=\operatorname{id}_{\mathbf{R}}$. Clearly $f$ is a continuous surjection. Since $\mathbf{R} \backslash \mathbf{Q}$ is dense in $\mathbf{R}$, it is nearly open but not open. 
The class of $B$-spaces may be of some interest, nevertheless, since it contains all locally compact Hausdorff spaces. It turns out that locally compact Hausdorff spaces play a peculiar role among $B$-spaces at least when we ask for the invariance of the class of $B$-spaces under sums.

PROPOSITION 2. Let $E$ be a completely regular space. Then the following statements are equivalent:

(1) For every locally compact Hausdorff space $L$ the sum $E \oplus L$ is a B-space.

(2) For every compact Hausdorff space $K$ the sum $E \oplus K$ is a $B$-space.

(3) $E \oplus \beta E$ is a $B$-space.

(4) $E$ is locally compact.

Proof. Clearly (1) implies (2) and (2) implies (3). Assume (3) and let $f: E \oplus$ $\beta E \rightarrow \beta E$ be defined by $f\left|E=e_{E}, f\right| \beta E=\operatorname{id}_{\beta E}$. Then $f$ is a continuous nearly open surjection and, consequently, is open. So $E$ is open in $\beta E$, hence is locally compact. Finally, assume (4) and let $f: E \oplus L \rightarrow F$ be a continuous, nearly open surjection onto the Hausdorff space $F$. Let $x \in E \oplus L$ and let $U$ be a compact neighborhood of $x$. Then $f(U)=\overline{f(U)}$, hence $f(x)$ is an interior point of $f(U)$ and so $f$ must be open.

REMARKS. (1) The situation for $B$-spaces is completely different from the $B_{r}$ case. For given a $B_{r}$-space $E$ and a locally compact Hausdorff space $L$ it is easy to see that $E \oplus L$ is again a $B_{r}$-space.

(2) Let $E$ be a nondiscrete Hausdorff Lindelöf $P$-space. Then

(a) $E \oplus E$ is a $B$-space (since it is Lindelöf $P$ );

(b) $E \oplus \beta E$ is not a $B$-space (since $E$ is not locally compact).

This proves that a completely regular space containing a dense subspace which is a $B$-space need not be a $B$-space itself, i.e., the class of $B$-spaces is not closed with respect to the operation of taking Hausdorff extensions. This is once more a surprising fact, since the corresponding result is true for $B_{r}$-spaces.

THEOREM 3. Let $E$ be a Hausdorff semiregular space containing a dense subspace $F$ which is a $B_{r}$-space. Then $E$ is itself a $B_{r}$-space.

ProOF. Let $f: E \rightarrow G$ be a continuous, nearly open bijection onto the Hausdorff space $G$. Then the restriction $f \mid F: F \rightarrow f(F)$ is continuous and bijective and moreover is nearly open. So we have $F \cong f(F)$. Now fix $x \in E$ and a neighborhood $U$ of $x$. Choose a regular-open neighborhood $V$ of $x$ having $V \subset U$. We prove int $\overline{f(V)} \subset f(U)$. Let $y \in \operatorname{int} \overline{f(V)}, y=f(z)$. Let $W$ be an open neighborhood of $z$ with $f(W) \subset$ int $\overline{f(V)}$. It is sufficient to prove $W \subset \bar{V}$. So let $w \in W$ and some regular-open neighborhood $O$ of $w$ having $O \subset W$ be fixed. It remains to prove $O \cap V \neq \varnothing$.

$O, V$ being regular-open in $E, O \cap F$ and $V \cap F$ are regular-open in $F$ and consequently $f(O \cap F)$ and $f(V \cap F)$ are regular-open in $f(F)$. On the other hand, the sets int $\overline{f(O)} \cap f(F)$, int $\overline{f(V)} \cap f(F)$ are regular-open sets in $f(F)$ densely containing the previous ones. This implies $f(O) \cap f(F)=\operatorname{int} \overline{f(O)} \cap f(F), f(V) \cap$ $f(F)=\operatorname{int} \overline{f(V)} \cap f(F)$. Since $O \subset W$ yields int $\overline{f(O)} \subset \operatorname{int} \overline{f(V)}$, hence $f(O) \cap$ $f(F) \subset f(V)$, we obtain the desired relation $O \cap V \neq \varnothing$.

We may derive the following result which we have obtained in $\left[\mathbf{N}_{1}\right.$, Theorem 1] by a direct argument. 
COROLLARY. Every Hausdorff semiregular space $E$ containing a dense Čech complete subspace is a $B_{r}$-space.

PROOF. This follows from Theorem 3 and the fact, proved in [BP], that every Čech complete space is a $B_{r}$-space.

\section{BIBLIOGRAPHY}

[BP] T. Byczkowski and R. Pol, On the closed graph and open mapping theorems, Bull. Acad. Polon. Sci. 24 (1976), 723-726.

[Ca] D. E. Cameron, Maximal and minimal topologies, Trans. Amer. Math. Soc. 160 (1971), 229248.

[E] R. Engelking, General topology, Monografie Matematyczne, vol. 60, PWN, Warsaw, 1977.

[FK] W. G. Fleissner and K. Kunen, Barely Baire spaces, Fund. Math. 101 (1978), 229-240.

[Gr] D. L. Grant, Topological groups which satisfy an open mapping theorem, Pacific J. Math. 68 (1977), 411-423.

[Kö] G. Köthe, Topological vector spaces. II, Grundlehren Math. Wiss., vol. 237, Springer-Verlag, Berlin and New York, 1979.

[Lu] D. J. Lutzer, On generalized ordered spaces, Dissertationes Math. 89.

$\left[\mathrm{N}_{1}\right]$ D. Noll, Open mapping theorems in topological spaces, Czechoslovak. Math. J. 35 (110), 1985.

$\left[\mathbf{N}_{2}\right] \ldots$, Sums and products of $B_{r}$-spaces, Pacific J. Math. 123 (1986), 173-188.

[Su] L. J. Sulley, A note on $B$ - and $B_{r}$-complete topological Abelian groups, Proc. Cambridge Philos. Soc. 66 (1969), 275.

[V] M. Valdivia, $B_{r}$-complete spaces which are not B-complete, Math. Z. 185 (1984), 253-259.

[We] J. D. Weston, On the comparison of topologies, J. London Math. Soc. 32 (1957), 342-354.

Universität StUttgart, Mathematisches Institut B, PfaffenWaldring, 7000 STUTTGART 80, BRD 